\title{
Co-complex caspase structures show unique interactions to highly specific probes
}

\author{
Dennis Willam Wolan ${ }^{1}$, Christopher Vickers ${ }^{1}$, Kim Angelo Solania ${ }^{1}$, Gonzalo González-Páez ${ }^{1}$ \\ ${ }^{1}$ The Scripps Research Institute, La Jolla, United States \\ E-mail: wolan@scripps.edu
}

We know surprisingly little about the roles of individual caspases in cell death despite members of this protease family having been associated with apoptosis for over two decades. The current compendium of caspase peptide inhibitors and substrates are highly promiscuous and do not provide the resolution required to assign any current cell-based observations to individual caspases, whereas genetically engineered systems offer tenuous clues to caspase function but only in somewhat artificial settings. For example, the highly homologous executioner caspases-3 and -7 that are assumed to target conserved biological substrates during the final stages of apoptosis are indistinguishable with respect to their canonical recognition peptide sequence, DEVD. However, knockout mouse models of either caspase-3 or -7 harbor divergent phenotypes and studies employing cell-free lysates indicate these two caspases may have distinct cellular targets. Thus, there is an urgent need to decipher the roles of specific caspases within cells and to control their activities. We employed a new methodology incorporating unnatural amino acids and novel warheads to identify first-in-class cell-permeable peptide-based inhibitors and substrates for caspase- 3 with 100x selectivity over caspase- 7 and all other caspases. Likewise, we have extended this method to the design of peptide probes specific for initiator caspases-8 and -9 with $>100$-fold selectivity over executioner caspases-3, -6 , and -7 . X-ray structures of caspases in complex with the tailored peptides are critical to the design and iterative improvement of our probes and provide mechanistic insights as to why and how our molecules generate specificity to their target protease. We show that incorporation of unnatural amino acids into peptide sequences exploits side chain interactions unique to each caspase active site. Specifically, addition of a 3-pyridylalanine onto the caspase-3 probe at position P5 exploits active site serine and asparagine residues found only within the active site of caspase-3 (PDB ID: 4JJE) in comparison to caspase-7 (PDB ID: 4JJ8) and caspase-8 (PDB ID: 4JJ7). Similarly, addition of a hydroxyproline in our caspase- 8 and -9 probe at position P2 is readily accommodated by these initiator caspases (PDB ID: P4S1); however, the presence of large hydrophobic side chains in the active sites of executioner caspases result in van der Waals repulsion against this unnatural amino acid (PDB ID: P4S0). Overall, our structures demonstrate many caspase-specific active-site interactions not previously identified that likely play significant roles in the recognition and hydrolysis of proteins unique to individual human caspase family members. Our efforts demonstrate that our design paradigm and validation methods are generally applicable to all caspases and can be extended to include other proteolytic enzymes.

[1] Vickers, C.J. et al. (2013) ACS Chem. Biol. 8, 1558-1566.

[2] Vickers, C.J. et al. (2013) J. Amer. Chem. Soc. 135, 12869-12876.

[3] Vickers, C.J. et al. (2014) ACS Chem. Biol. 9, 2194-2198.

Caspase-3

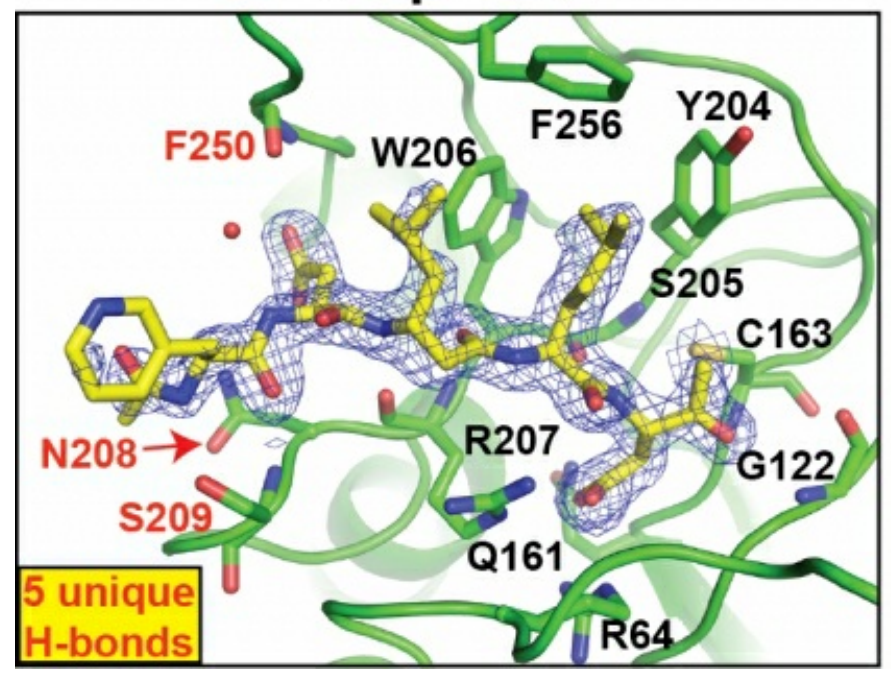

Caspase-7

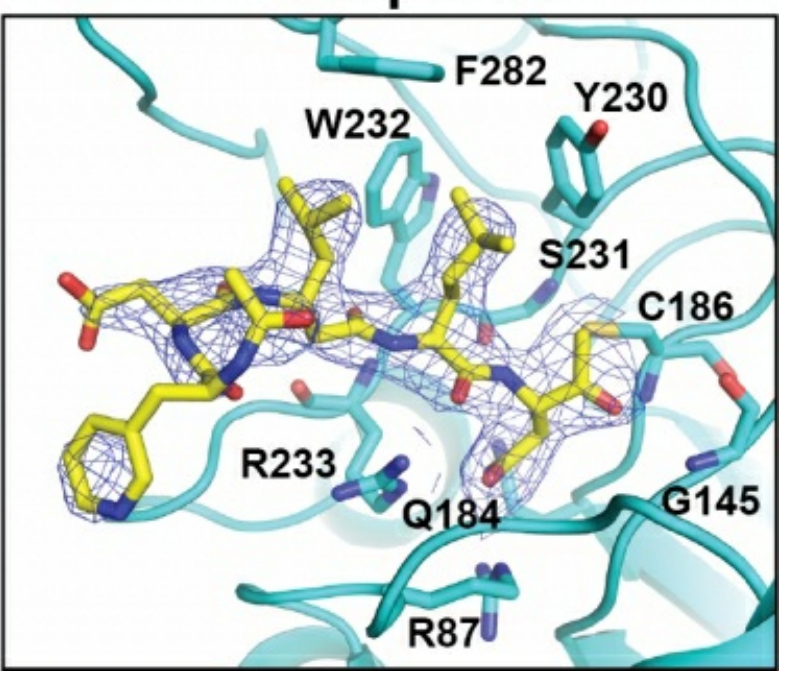

Keywords: caspase, probes, peptides 